\title{
Una Propuesta de Reconstrucción del Significado Holístico de la Antiderivada
}

\author{
A Proposal for Reconstruction of the Holistic Meaning of the Anti- \\ derivative
}

\author{
Wilson Gordillo* \\ Luis R. Pino-Fan ${ }^{* *}$
}

\begin{abstract}
Resumen
En el presente estudio se informan los resultados obtenidos al analizar diversas situaciones-problemas que fueron abordados a lo largo de la historia, y que permitieron el surgimiento y evolución de la noción antiderivada. Dichas situaciones-problema, y sus soluciones, fueron identificadas mediante un estudio histórico-documental, y analizados mediante el uso de algunas de las herramientas, teórico-metodológicas, que nos proporciona el marco teórico conocido como Enfoque Onto-semiótico (EOS) del conocimiento y la instrucción matemáticos. Como resultado, se encontró que a lo largo de la historia la antiderivada ha adoptado cuatro significados parciales, que en conjunto, conforman una propuesta de reconstrucción del significado holístico de referencia para dicha noción.
\end{abstract}

Palabras-clave: Antiderivada. Estudio histórico-epistemológico. Enfoque ontosemiótico. Significado holístico. Configuración epistémica.

\begin{abstract}
The present study reports the results obtained by analysing different problem-situations addressed throughout history and which allowed both the emergence and evolution of the anti-derivative notion. Such problemsituations and their solutions were identified by a historical documentary study and analysed by using some of the methodological-theoretical tools, which provided the theoretical framework known as Onto-Semiotic Approach (OSA) of mathematical knowledge and instruction. As a result, it was found that, throughout history, the anti-derivative notion adopted four partial meanings that together comprised a proposal for reconstruction of the holistic meaning for this notion.
\end{abstract}

Keywords: Anti-derivative. Historical-epistemological study. Onto-semiotic approach. Holistic meaning. Epistemic configuration.

\section{Introducción}

El estudio histórico-epistemológico de los objetos matemáticos, según Anacona

\footnotetext{
"Estudiante del Doctorado en Educación Matemática de la Universidad de Los Lagos (ULAGOS). Profesor Asociado de la Universidad Distrital Francisco José de Caldas (UDFJC), Bogotá, Colombia. Dirección postal: Carrera 7 No. 40-53, Barrio Sucre, 110231, Bogotá, Colombia. E-mail: wgordillot@udistrital.edu.co

*** Doctor en Didáctica de la Matemática por la Universidad de Granada (UGR). Profesor del Departamento de Ciencias Exactas de la Universidad de Los Lagos (ULAGOS), Santiago, Chile. Dirección postal: Av. República, 517, Barrio República, 8370341, Santiago, Chile. E-mail: luis.pino@ulagos.cl
} 
(2003), aporta elementos que refieren a las distintas concepciones de los objetos matemáticos, en los que se analizan las posibles dificultades que se presentaron en la construcción de éstos. De acuerdo con D’Ambrosio (2013), la comprensión de los objetos matemáticos depende de la comprensión de cómo se originan y de sus motivaciones para el desarrollo.

En este trabajo realizamos un estudio histórico-epistemológico de la antiderivada, con el fin de determinar el origen de este objeto matemático. El recuento histórico-documental, muestra la relación existente entre la antiderivada y otros dos objetos matemáticos, fundamentales para el análisis matemático, a los que está vinculada: la derivada y la integral. La derivada y la integral han sido objeto de estudios histórico-epistemológicos, cada uno de forma independiente. Para la derivada, Mateus (2011); Cantoral (2000); Pino-Fan, Godino y Font (2011) y Pino-Fan (2011). Por su parte, para la integral, Cordero (2002); Cantoral y Farfán (2004); Cabañas-Sanchez (2011) y Crisóstomo (2012).

El estudio que realizamos, toma como base la relación entre estos dos objetos matemáticos, y por ende, los aportes de los estudios histórico-epistemológicos citados anteriormente - sobre la integral y la derivada -, con el fin de determinar cómo emerge la antiderivada, la cual ejerce el rol de mediador entre el cálculo diferencial y el cálculo integral a través de lo que conocemos hoy en día como el Teorema Fundamental del Cálculo (TFC).

El objetivo de este trabajo es presentar una propuesta de reconstrucción del significado holístico de referencia para la antiderivada. Con esta finalidad, presentamos el resultado de un estudio histórico-epistemológico de tipo documental, que hemos comentado, el cual se realizó con el fin de identificar las diversas etapas históricas de las cuales emerge dicho objeto matemático. A través de situaciones-problemas abordados por los matemáticos de la época, describimos las características de las prácticas desarrolladas en diversos momentos históricos, los cuales contribuyeron al surgimiento y formalización de la antiderivada como objeto matemático. Para el análisis de las prácticas matemáticas desarrolladas, utilizamos algunas de las herramientas que nos proporciona el Enfoque Onto-Semiótico (EOS) del conocimiento y la instrucción matemática. Concretamente, utilizamos la noción de configuración epistémica, la cual nos permitió identificar y caracterizar cuatro significados parciales para la antiderivada, los cuales conforman el significado holístico.

\section{Marco teórico y metodología}

Para esta investigación hemos utilizado el marco teórico conocido como Enfoque Onto-semiótico (EOS) del conocimiento matemático, desarrollado en diversos trabajos por 
Godino y colaboradores (GODINO; BATANERO, 1994; GODINO; BATANERO, 1998; GODINO; BATANERO; FONT, 2007). Este marco teórico incluye un modelo epistemológico sobre las matemáticas, construido sobre bases antropológicas y socioculturales, un modelo cognitivo, sobre bases semióticas de índole pragmatista.

Algunas de las bases de construcción del EOS, se operativizan a través de nociones que actúan como herramientas al analizar un objeto matemático. Entre ellas se tiene la noción de sistema de prácticas descrita como "toda actuación o manifestación (lingüística o no) realizada por alguien para resolver problemas matemáticos, comunicar a otros la solución, validar la solución y generalizarla a otros contextos y problemas" (GODINO; BATANERO, 1994, p. 334).

En este estudio en particular, utilizamos la relación objeto matemático y sistema de prácticas. En el EOS, los objetos matemáticos son considerados como entidades emergentes de los sistemas de prácticas realizados para resolver un campo de problemas (GODINO; BATANERO, 1994). En las prácticas matemáticas intervienen objetos ostensivos (símbolos, gráficos etc.) y no ostensivos (conceptos, proposiciones etc.) (PINO-FAN, 2014), que rememoramos al hacer matemáticas y que son representados en forma textual, oral, gráfica o incluso gestual. De los sistemas de prácticas matemáticas operativas y discursivas emergen nuevos objetos que provienen de las mismas y dan cuenta de su organización y estructura. En este sentido, dentro del EOS se propone la siguiente tipología de objetos matemáticos primarios, que intervienen en los sistemas de prácticas (GODINO; BATANERO; FONT, 2007):

- Elementos lingüísticos (términos, expresiones, notaciones, gráficos...) en sus diversos registros (escrito, oral, gestual...).

- Situaciones/Problemas (aplicaciones extra-matemáticas, ejercicios...).

- Conceptos/Definiciones (introducidos mediante definiciones o descripciones) (recta, punto, número, media, función, derivada...).

- Proposiciones/Propiedades (enunciados sobre conceptos...).

- Procedimientos (algoritmos, operaciones, técnicas de cálculo...).

- Argumentos (enunciados usados para validar o explicar las proposiciones y procedimientos, deductivos o de otro tipo...).

Los seis objetos matemáticos anteriores, conforman lo que denominaremos configuración ontosemiótica, la cual puede ser de tipo cognitiva (configuración cognitiva) si se tratan de los objetos matemáticos primarios que moviliza un sujeto como parte de su 
práctica matemática desarrollada a propósito de la solución de un problema, o de tipo epistémica (configuración epistémica) si se trata de los objetos matemáticos institucionales. En este trabajo utilizamos la noción de configuración ontosemiótica en su versión epistémica (Figura 1), o configuración epistémica, (PINO-FAN; GODINO, FONT, 2011), puesto que nos ayuda a determinar el significado global, también denominado significado holístico u holosignificado, el cual comprende los diferentes significados parciales de un objeto matemático (PINO-FAN; GODINO; FONT, 2011; PINO-FAN, 2014).

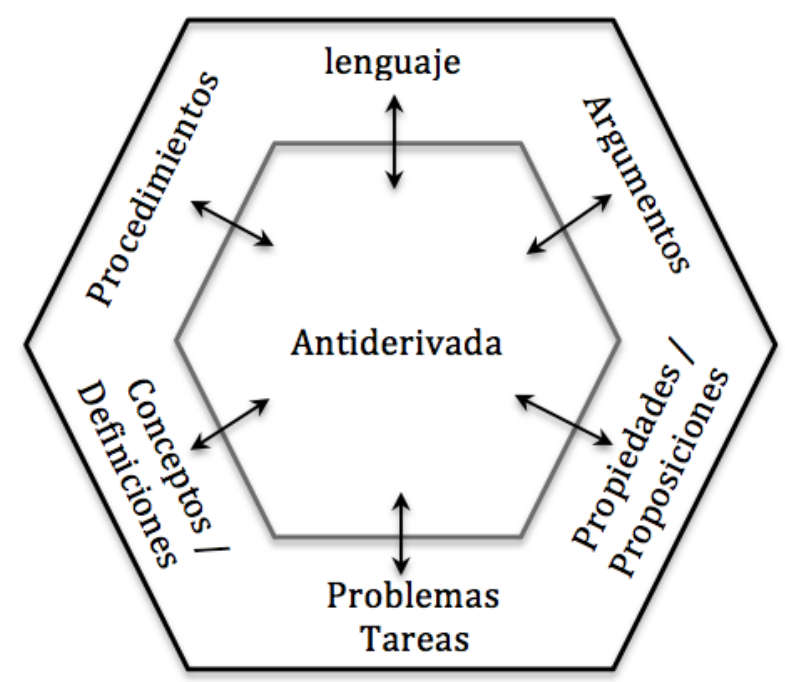

Figura 1- Configuración epistémica de la antiderivada

Esta noción de configuración epistémica, nos permitió analizar y describir sistemáticamente los objetos matemáticos primarios, y sus significados, que intervienen en las prácticas matemáticas de la antiderivada, de acuerdo con su etapa histórica.

\section{Un estudio histórico-epistemológico sobre la antiderivada}

A continuación se presenta un estudio histórico-epistemológico, de tipo documental, que tiene por objetivo la identificación de las principales problemáticas que fueron abordadas en distintas etapas históricas y que dan origen a la emergencia y evolución del objeto matemático antiderivada. Como referente inicial, consideramos aquellas problemáticas en las que se contempla, explícita o implícitamente, la relación entre la derivada y la integral.

En este sentido, se ha dividido este estudio en cuatro grandes bloques: 1) la génesis de la antiderivada; 2) la antiderivada en la Época Medieval; 3) la evolución de la antiderivada durante el siglo XVII; y 4) en busca del rigor y la fundamentacion matemática de la antiderivada. Cada uno de los cuatro bloques mencionado, refiere a una problemática 
abordada por matemáticos de la época.

\subsection{La génesis de la antiderivada}

Los matemáticos griegos se destacaron en sus procedimientos geométricos intuitivos, en especial, se dedicaron a encontrar tangentes y cuadraturas de diferentes superficies. Sus trabajos son la base de la génesis de la antiderivada, por esto es importante señalar a uno de ellos en particular, quien trabaja con las dos nociones matemáticas que vinculan a dicho objeto. Fue Arquímedes de Siracusa (287 a.C.-212 a.C.), quien en sus trabajos utiliza la noción de recta tangente y sus propiedades, para el trazado de polígonos circunscritos a circunferencias; dicha noción fue propuesta años antes por Euclides de Alejandría (325 a.C265 a.C.). En un Palimpsesto ${ }^{l}$ encontrado a principios del siglo XX y analizado con técnicas de imagen multiespectral, en 1998, se describen evidencias de propuestas hechas por Arquímedes para encontrar geométricamente la tangente a una curva dada, la cual lleva su nombre: la espiral de Arquímedes. La descripción, hecha por Boyer (1999), señala que los matemáticos griegos, entre ellos Arquímedes, no disponían de una definición muy satisfactoria de tangente a una curva $C$ en un punto $P$, considerándola como una recta $L$ que tiene el único punto $P$ común con la curva, de forma que no puede trazarse ninguna otra recta que pase por $P$ que este incluida entre la recta $L$ y la curva $C$. De acuerdo con Pino-Fan (2011), es posible que Arquímedes tuviera que romper con esta concepción para trazar la tangente de la espiral, adoptando un punto de vista cinemático que le permitió determinar la dirección instantánea del movimiento del punto mediante el cual se genera la curva.

Con respecto a la cuadratura, fue Arquímedes quien a través de la descomposición de polígonos en triángulos, como se muestra en su obra Quadrature of the parabole (cuadratura de la parábola), demuestra geométricamente que un segmento de parábola puede agotarse ${ }^{2}$ mediante una serie de triángulos de área $A$, tal como se muestra en la Figura 2. Este procedimiento lo llevó a demostrar que el área del segmento parabólico es

$$
A\left(1+\frac{1}{4}+\frac{1}{4^{2}}+\cdots+\frac{1}{4^{n-1}}+\frac{1}{3} \cdot \frac{1}{4^{n-1}}\right)=\frac{4}{3} A
$$

\footnotetext{
${ }^{1}$ Texto antiguo escrito sobre otro anterior en papiro, formando un libro o códice.

${ }^{2}$ Procedimiento geométrico de aproximación en el cual se inscriben triángulos en una curva. Este procedimiento también es conocido como método de exhaución.
} 


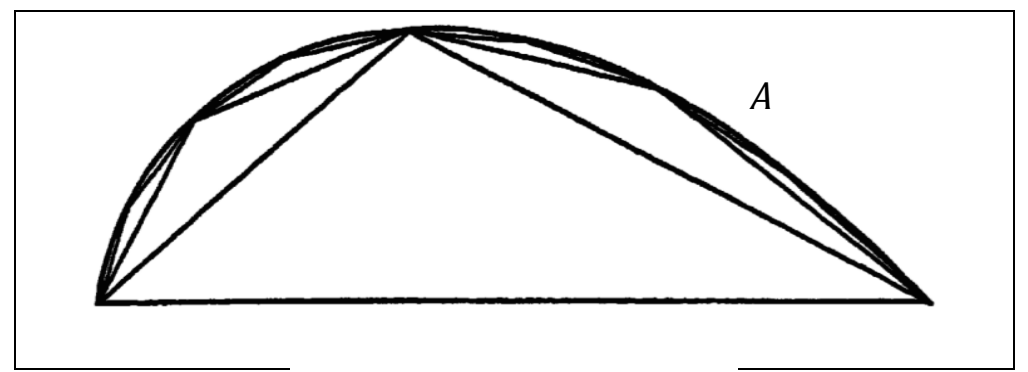

Figura 2- Método de exhaución

Si bien, este método de demostración es geométrico, permite determinar áreas y volúmenes sin hacer uso de límites.

En esta etapa histórica aparecen las tangentes - proto derivadas (PINO-FAN, 2011) y las cuadraturas (integrales), como dos objetos matemáticos emergentes de prácticas geométricas. No hay evidencia que indique el estudio, en este período, de la relación de dichos objetos. Se tuvo que esperar hasta el medioevo para ver nuevos aportes en cuanto a esta relación.

\subsection{La antiderivada en la época medieval}

Los antecedentes planteados por los griegos, y en especial por Arquímedes, influyeron en los matemáticos de la Época Medieval, entre ellos Isaac Barrow (1630-1677), quien en su obra Lectiones Opticae et Geometricae (Lecciones de Óptica y Geometría), destacó la relación geométrica entre las tangentes y las cuadraturas (Figura 3). En una de las proposiciones de la lección $\mathrm{X}$ de dicha obra, describe:

Sea ZGE una curva cuyo eje es VD y consideremos las ordenadas (VZ, PG y DE) perpendiculares a este eje y continuamente creciendo desde la ordenada inicial VZ; también sea VIF una curva tal que si una línea recta EDF es trazada perpendicular al eje VD, cortando a las curvas en los puntos $\mathrm{E}, \mathrm{F}$ y $\mathrm{VD}$ en $\mathrm{D}$, el rectángulo determinado por DF y una longitud dada $\mathrm{R}$ es igual al espacio VDEZ; también sea $\mathrm{DE}: \mathrm{DF}=\mathrm{R}: \mathrm{DT}$, y unimos [T y F]. Entonces TF cortará a la curva VIF. Tomemos un punto I en la curva VIF (primero del lado F hacia V) y, a través de él, tracemos IG paralelo a VZ y IL paralelo a VD, cortando a las líneas dadas como se muestra en la figura [Figura 3.2]; entonces, $\mathrm{LF}: \mathrm{LK}=\mathrm{DF}: \mathrm{DT}$, es decir $\mathrm{R} \times \mathrm{LF}=\mathrm{LK} \times \mathrm{DE}$. Pero de la naturaleza de las líneas DF y LK se tiene $\mathrm{R} \times \mathrm{LF}=$ área $(\mathrm{PDEG})$, por tanto se tiene que $\mathrm{LK} \times \mathrm{DE}=$ área $(\mathrm{PDEG})<\mathrm{D} \times \mathrm{DE}$, por lo tanto se tiene $\mathrm{LK}<\mathrm{DP}<\mathrm{LI}$. De forma análoga, si el punto I se toma del otro lado de F, se haría la misma construcción de antes y se puede fácilmente demostrar que LK $>$ DP $>$ LI. A partir de lo anterior, es completamente claro que toda línea TKF permanece en o debajo de la curva VIF. Resultados análogos se obtienen si las ordenadas VZ, PG y DE decrecen en forma continua, la misma conclusión se obtiene mediante un argumento similar. Sólo una particularidad ocurre, a saber, en este caso, al contrario que en el otro, la curva VIF es cóncava respecto al eje VD (BARROW, 1735, p. 167). 


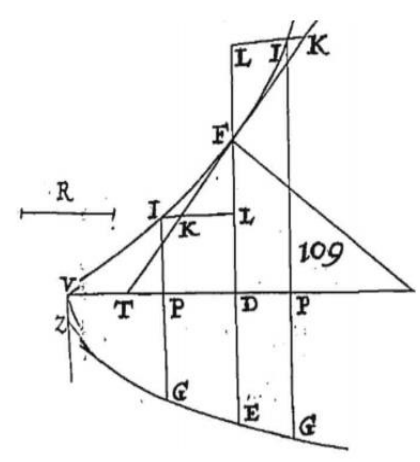

Figura 3- Diagrama de Barrow Fuente: BARROW, 1735, p. 167

La proposición anterior establece que para trazar una recta tangente a una curva, ésta última debe estar relacionada con la cuadratura de otra curva. Este resultado geométrico es asumido en la actualidad como la versión preliminar del TFC.

En esta etapa histórica, el objeto matemático antiderivada emerge con procedimientos geométricos que relacionan la tangente de una curva con su cuadratura. El argumento de dicha relación (las tangentes con sus cuadraturas) se basa en construcciones geométricas planteadas por los griegos.

Barrow, tal como se mostró anteriormente, avanza al poder establecer la relación geométrica entre la tangente de una curva y su cuadratura, relación que hasta esa época no había sido establecida. Este gran avance de Barrow, hizo detonar en los matemáticos de la época, y en algunos de sus discípulos, una serie de aportes no geométricos en relación con la derivada, la integral, y la relación entre ambas nociones.

\subsection{La evolución de la antiderivada durante el siglo XVII}

Al final de la época barroca, en el siglo XVII, Sir Isaac Newton (1642-1727) y Gottfried Wilhelm Leibniz (1646-1716), tomaron las bases teóricas matemáticas heredadas de Kepler, Cavalieri, Torricelli, Fermat, Roberval, Barrow, entre otros, y cada uno de forma independiente, y de acuerdo con Pino-Fan (2014), fundaron lo que hoy denominamos cálculo infinitesimal. Según Collete (1985), los predecesores de Newton y Leibniz habían utilizado métodos de análisis para resolver problemas con curvas especificas, algunas de los cuales ya habían sido abordados por los matemáticos griegos. El primero de estos problemas fue: dada la fórmula para la distancia recorrida por un cuerpo como función del tiempo, encontrar la velocidad y aceleración instantánea. E inversamente: dada la fórmula para la aceleración como una función del tiempo, encontrar la velocidad y la distancia recorrida. El segundo 
problema trataba sobre la búsqueda de la tangente a una curva dada en un punto dado (problema de las tangentes). El tercer problema se trataba sobre la búsqueda analítica de valores máximos y mínimos de una función. Por último, el cuarto problema consistía en encontrar el área y el volumen acotados por curvas y superficies, respectivamente (problema de las cuadraturas). De acuerdo con Ponce (2013), los problemas antes mencionados fueron abordados generalmente, como casos aislados.

\title{
3.3.1 Newton: el aporte cinemático
}

En el libro The methodus of fluxions and infinite series (Método de fluxiones y series infinitas), Newton concibe las cantidades matemáticas como el movimiento continuo de un punto que traza una curva:

\begin{abstract}
Llamaré cantidades fluentes, o simplemente fluentes, a estas cantidades que considero aumentadas gradualmente e indefinidamente; las representaré mediante las últimas letras del alfabeto $\mathrm{v}, \mathrm{x}, \mathrm{y}, \mathrm{z}$, para distinguirlas de las otras cantidades que, en las ecuaciones, se consideran como conocidas y determinadas, y que se representan por las primeras letras a, b, c, etc. Representaré con las mismas últimas letras coronadas con un punto $\dot{\mathrm{v}}, \dot{\mathrm{x}}, \dot{\mathrm{y}}, \dot{\mathrm{z}}$, las velocidades con que las fluentes aumentan por el movimiento que las produce y que, por consiguiente, se pueden llamar fluxiones (NEWTON, 1736, p. 20).
\end{abstract}

Newton introduce, inicialmente, la noción de fluxiones de forma geométrica, en curvas cinemáticas que describen comportamientos en función del tiempo. Newton describe el movimiento de un punto como la trayectoria de un móvil, en donde la velocidad en cada punto tiene componentes, según las direcciones de los ejes $\dot{x}, \dot{y}$. Para hallar la pendiente de la recta tangente a la curva dada en un punto, calcula el cociente $\frac{\dot{y}}{\dot{x}}$ de direcciones de los ejes. Este cociente de diferenciación, para la época, era sencillo, tanto que en los escritos de Newton, se escriben tablas que le posibilitan resultados directos para este cociente; la finalidad de estas tablas era la minimización de esfuerzos matemáticos en la búsqueda de las propiedades de las curvas conocidas en su tiempo. Al mismo tiempo, Newton planteó el problema inverso: dado el cociente $\frac{\dot{y}}{\dot{x}}$, cómo encontrar $y$ en función de $x$.

Newton abordó el desarrollo del cálculo a partir de la geometría analítica, desarrollando un enfoque geométrico y analítico de lo que hoy conocemos como derivadas, las cuales aplicó sobre curvas definidas a través de ecuaciones. Newton también buscaba cómo cuadrar distintas curvas, y la relación entre la cuadratura y la teoría de tangentes, convirtiéndose este en un problema fundamental para él: dada una relación entre fluentes, encontrar la correspondiente relación entre sus fluxiones; y de forma recíproca, dada una 
relación entre fluxiones, hallar la correspondiente relación entre fluentes.

Newton se percató de que el método de fluxiones podía utilizarse para obtener las velocidades instantáneas de una trayectoria conocida. En sus primeras investigaciones trabajó únicamente con problemas geométricos, como encontrar tangentes, curvaturas y áreas, utilizando como base matemática la geometría analítica de Descartes. No obstante, con el afán de separar su teoría de la de Descartes, comenzó a trabajar únicamente con las ecuaciones y sus variables, sin necesidad de recurrir al sistema cartesiano. Por ejemplo, la aproximación del cálculo del área (Figura 4), la describe en su obra Philosophiae Naturalis Principia Mathematica (principios matemáticos de la filosofía natural), tal como se detalla en la siguiente proposición:

En una forma curva, son rectas Aa y AE, y acE comprende la curva. En ella [la
curva] se inscriben un número infinito de paralelogramos $\mathrm{Ab}, \mathrm{Bc}, \mathrm{Cd}, \ldots$, con bases
$\mathrm{AB}, \mathrm{BC}, \mathrm{CD}, \ldots$, iguales, y los lados $\mathrm{Bb}, \mathrm{Cc}, \mathrm{Dd}, \ldots$, mantenidos en paralelo al lado
de la recta aA y completando los paralelogramos aKbl, bLcm, cMdn,... La amplitud
de dichos paralelogramos podría disminuir y su número ser aumentado hasta el
infinito; es decir, que las razones últimas están relacionadas entre sí, a su vez, la
figura inscrita AKbLcMdD, la figura circunscrita AalbmcndoE, y la figura
curvilínea AabcdE, son razones iguales (NEWTON, 1686, p. 25).

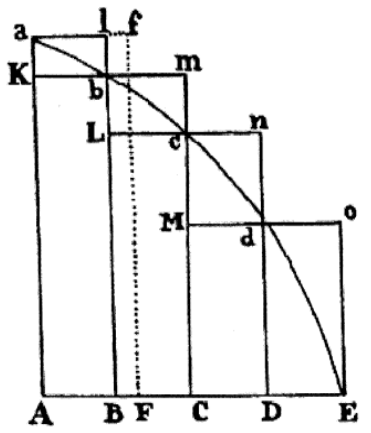

Figura 4- Aproximación de área Fuente: NEWTON, 1686, p. 25)

Newton introduce un lenguaje al referirse a fluxiones y fluentes, en la concepción de dos problemas; el primero consistió en encontrar la velocidad del movimiento en un tiempo dado cualquiera, dada la longitud del espacio descrito. El segundo problema es planteado como el reciproco del primero. Esta relación, cinemática, entre las fluxiones y las fluentes, evidencian el surgimiento de proto-antiderivada, la cuál para Newton es, originalmente, una integral indefinida. Newton también incluyo en su obra sobre fluxiones tablas de curvas clasificadas, que comprendían regiones limitadas por la abscisa y la ordenada para cada una de las formas curvas. Estas tablas corresponden en la actualidad a reglas de integración para el cálculo de áreas. También incluye el desarrollo de series infinitas que son aprovechadas años más tarde en el cálculo infinitesimal. 


\subsubsection{Leibniz: el aporte de análisis matemático}

En el mismo período, como lo indicamos anteriormente y de acuerdo con Collete (1985), Leibniz elabora un enfoque basado en el concepto de sumas y diferencias finitas (caso discreto) y, cuando se aplica a curvas (caso continuo), pueden llegar a ser infinitamente pequeñas. Su finalidad era elaborar un método eficaz mediante el cual, sin recurrir a diagramas, ciertas propiedades de las curvas puedan ser determinadas por medio de su "cálculo de las diferencias" (PINO-FAN, 2014, p. 96). Sus ideas permiten interpretar el paso de lo intuitivo a lo formal, con una construcción simbólica, lo que representa un gran avance al desarrollo del calculo infinitesimal. Leibniz, al igual que Newton, utilizó un nuevo lenguaje notacional, tal como se evidencia en su trabajo con el símbolo $\int l$ (suma de todas las $l$ ), para generar la sustitución de la notación usada por Cavalieri para la misma suma, "Omn $l$ " (suma de todas las $l$ ). En sus manuscritos describe que el símbolo integral $\int$, corresponde a la forma de la letra $S$ alargada, que se deriva del latín summa, lo que indicaba cada vez que realizaba una suma de valores sucesivos. Así mismo, introduce el símbolo $d$ para referirse a los diferenciales, este símbolo corresponde a la letra $d$, que se deriva del latín differentia, lo que indicaba cada vez que realizaba diferencias de valores sucesivos finitos. Esta notación es, probablemente, el legado más perdurable en el cálculo, puesto que sigue vigente hasta nuestros días.

Su primera publicación, en 1684, aparece en el Acta Eruditorum, y la tituló Nova methodus pro maximis et minimis, intemque tangetibus, qua nec irrationales quantitates moratur (Nuevo método para los máximos y mínimos, así como para las tangentes, el cual puede también aplicarse a las cantidades fraccionarias e irracionales). En esta obra, según Boyer (1959), aparece la definición de diferencial o cantidad diferencial, así como algunas reglas, sin pruebas, de diferenciales de sumas, productos, cocientes, máximos y mínimos, y puntos de inflexión.

Los argumentos propuestos por Leibniz, descritos por Boyer (1959), muestran el manejo de propiedades para determinar el área bajo una curva, la vinculación de límites de sumas en funciones continuas con la integral definida, llevó a Leibniz a descubrir lo que hoy se conoce como el TFC, como se muestra a continuación:

Si $F(x)=\int_{a}^{x} f(x) d x$, para una función continua $f(x)$, tiene una derivada que es la misma, $F^{\prime}(x)=f(x)$. En general, es posible encontrar valores para $x=a, x=b$, de un intervalo [a, b] donde la función $F(x)$ puede ser derivada de $f(x)$. La función $F(x)$, llamada primitiva de $\mathrm{f}(\mathrm{x})$, y los valores $\mathrm{F}(\mathrm{b})-\mathrm{F}(\mathrm{a})$, son ocasionados por la definición de $\int_{\mathrm{a}}^{\mathrm{b}} \mathrm{f}(\mathrm{x}) \mathrm{dx}$, en el caso que la relación 
$\int_{a}^{\mathrm{x}} \mathrm{f}(\mathrm{x}) \mathrm{dx}=\lim _{\mathrm{n} \rightarrow \infty} \sum_{\mathrm{i}=1}^{\mathrm{n}} \mathrm{f}\left(\mathrm{x}_{\mathrm{i}}\right) \Delta \mathrm{x}_{\mathrm{i}}$ es el teorema fundamental del calculo, que define la integral definida (BOYER, 1959, p. 11).

La descripción de Leibniz, relaciona el objeto derivada y la integral definida, a través de una función llamada primitiva o antiderivada.

En términos comparativos, de los trabajos y desarrollos de los fundadores del cálculo (Newton y Leibniz), se podría decir que el trabajo de Newton se basa en variaciones respecto al tiempo, pues el origen de sus ideas, tal como lo hemos comentado, es físico. Leibniz, por su lado, parte de problemas filosóficos buscando infinitesimales, de ahí que su trabajo se base en sumas de infinitesimales, sumas que son asumidas como integrales definidas y para las cuales requiere el uso de funciones primitivas (antiderivadas) para el cálculo respectivo de áreas.

\subsection{En busca del rigor y la fundamentacion matemática de la antiderivada}

Posterior a los desarrollos de Newton y Leibniz, el estudio y desarrollos relativos al cálculo infinitesimal, continúa con los hermanos Bernoulli. El primero de ellos, Jacques Bernoulli (1654-1705) fue quien utilizó, por primera vez, la expresión integral, en su obra sobre la isócrona publicada en el Acta Eruditorum, de 1690. Anteriormente, él había sugerido esta expresión a Leibniz, quien a su vez utilizó la expresión calculus integralis (cálculo integral) en sustitución de calculus summatorius (cálculo sumatorio) para referirse al proceso inverso del calculus differentialis (cálculo diferencial).

En 1742, el segundo de los hermanos, Jean Bernoulli (1667-1748) publicó en su Opera Omnia, el primer texto expositivo de cálculo integral, considerado satisfactorio por la Royal Society of London ${ }^{3}$. En dicha obra, considera la integral como la operación inversa de la diferencial con la adición adecuada de una constante, concepción distinta a la de Leibniz, quien consideraba la integral como una suma de cantidades infinitamente pequeñas. Fue Leonhard Euler (1707-1783) quien llevó, finalmente, a la noción matemática antiderivada a su desarrollo actual.

Euler fue quien planteó, para algunos problemas cinemáticos y geométricos, la solución sin necesidad de encontrar una función primitiva (antiderivada), a cambio propuso que se puede determinar una función desconocida a partir de una relación que la vincule con la derivada. Este proceso se describe en su tratado Institutionum calculi integralis. Volumen primun (Fundamentos de cálculo integral. Volumen primero). Euler consideró la incapacidad

\footnotetext{
${ }^{3}$ Sociedad científica del Reino Unido para el avance de las ciencias, fundada en 1660, para promover el saber experimental Físico-Matemático.
} 
de los métodos elementales propuestos por Leibniz y Newton para resolver problemas importantes, es decir, estos métodos se restringen a algunas funciones a las que denominó elementales ${ }^{4}$. Para dar solución a dichas funciones, introduce en su tratado un método numérico que permite solucionar cualquier situación, por ejemplo, si se considera a $f(x)=$ $e^{x^{2}}$, esta función es continua pero no puede ser expresada como una función elemental, por tal razón no tiene antiderivada, pero su integral sí existe y puede ser calculada. Al expresar la función por medio de una serie infinita de Maclaurin ${ }^{5}$, se tiene $e^{x^{2}}=\sum_{n=0}^{\infty} \frac{\left(x^{2}\right)^{n}}{n !}=1+\frac{x^{2}}{1 !}+$ $\frac{x^{4}}{2 !}+\frac{x^{6}}{3 !}+\cdots$, por lo tanto, $\int e^{x^{2}} d x=\int\left(1+\frac{x^{2}}{1 !}+\frac{x^{4}}{2 !}+\frac{x^{6}}{3 !}+\cdots\right) d x$, si se integra término a término se tiene $\int\left(1+\frac{x^{2}}{1 !}+\frac{x^{4}}{2 !}+\frac{x^{6}}{3 !}+\cdots\right) d x=C+x+\frac{x^{3}}{3 \cdot 1 !}+\frac{x^{5}}{5 \cdot 2 !}+\frac{x^{7}}{7 \cdot 3 !}+\cdots+\frac{x^{2 n+1}}{(2 n+1) \cdot n !}+$ ... Luego se determina si esta serie converge o diverge, a través de la regla de comparación ${ }^{6}$, para este caso se tiene que $\lim _{n \rightarrow \infty}\left|\frac{\frac{x^{2(n+1)}}{(n+1) !}}{\frac{x^{2 n}}{n !}}\right|=\lim _{n \rightarrow \infty}\left|\frac{n ! \cdot x^{2 n+2}}{(n+1) ! \cdot x^{2 n}}\right|=\lim _{n \rightarrow \infty}\left|\frac{x^{2}}{(n+1)}\right|$. Para cualquier valor que tome $x$, ésta se convierte en una constante, entonces se tiene que $\lim _{n \rightarrow \infty}\left|\frac{x^{2}}{(n+1)}\right|=0<$ 1, por lo tanto, la serie es convergente. Este método era utilizado por Newton en series de potencia. Hoy en día, esta forma de proceder se conoce como método de integración numérica.

Euler describe la relación entre el cálculo diferencial y el cálculo integral en la siguiente definición:

Definición 1: El cálculo integral es el método por el cual se encuentra la relación entre las cantidades de los diferenciales, la operación que se presenta aquí, es llamada integración.

Corolario 1: Por lo tanto, desde el cálculo diferencial se enseña a investigar la relación de los diferenciales de determinadas magnitudes de las variables; el cálculo integral es el método inverso (EULER, 1770, p. 3).

En este momento histórico, la noción matemática antiderivada alcanza su máximo desarrollo al demostrar que no todas las funciones tienen antiderivadas, tal como lo afirmó Euler, pero, sí, es posible calcular una integral definida sobre ellas, como se mostro anteriormente.

\footnotetext{
${ }^{4}$ Una función denominada elemental es una función construida a partir de una cantidad finita de funciones fundamentales y constantes, mediante operaciones (adición, sustracción, multiplicación y división) y/o la composición de funciones (NIKOLSKI, 1985, p.16).

${ }^{5}$ Caso especial de la serie de Taylor, llamada así en honor a Colin Maclaurin (1698-1746), quien la popularizó en su libro Treitase of Fluxions (Tratado de Fluxiones), en 1742.

${ }^{6}$ Prueba que, a través del limite al infinito, muestra la convergencia o divergencia de una serie de acuerdo con los siguientes criterios: a) si $\lim _{n \rightarrow \infty}\left|\frac{a_{n+1}}{a_{n}}\right|<1$, la serie es convergente; b) si $\lim _{n \rightarrow \infty}\left|\frac{a_{n+1}}{a_{n}}\right|>1$, la serie es divergente; y c) si $\lim _{n \rightarrow \infty}\left|\frac{a_{n+1}}{a_{n}}\right|=1$ no se puede concluir.
} 


\section{Configuraciones epistémicas asociadas a las problemáticas sobre antiderivadas}

El recorrido histórico-documental que realizamos anteriormente, permitió identificar diversas problemáticas que dieron paso a la emergencia de la antiderivada como objeto matemático. Dichas problemáticas las hemos catalogado en cuatro problemas fundamentales: a) el problema geométrico de las tangentes de una curva y la cuadratura de la misma; b) el problema de la relación fluxiones - fluentes; c) el problema sobre la relación de los diferenciales y las sumatorias; y d) el problema de la identificación de funciones elementales.

Cada uno de estos grandes problemas, refieren a sistemas de prácticas distintos que fueron abordados en diversas etapas históricas y se han analizados con las herramientas que nos proporciona el modelo teórico EOS.

A continuación, describimos el análisis de cada uno de los cuatro grandes problemas, comentados anteriormente, mediante el análisis de un ejemplo prototípico con el cual se caracterice la configuración epistémica de objetos matemáticos primarios asociada a determinado sistema.

\subsection{Configuración epistémica 1 (CE1): el problema geométrico de las tangentes y cuadraturas}

Este problema, descrito en el apartado 3.2 y mostrado geométricamente con la Figura 3, fue abordado por Barrow en la Época Medieval. En él, se puede observar la forma de construcción de una curva creciente, en la cual se traza una tangente en un punto dado, y por medio de construcciones geométricas se relaciona con la cuadratura de la curva dada. Este problema geométrico es prototípico tanto de las situaciones/problemas como de las prácticas matemáticas que hasta esa época se desarrollaban. Barrow logra unir dos conceptos separados hasta ese momento, la tangente a una curva y la cuadratura de la misma.

Las construcciones geométricas son un trabajo propio de los griegos y se utilizaron como único argumento, y lenguaje, hasta el siglo XVII. Todas las propiedades y procedimientos están apoyadas en construcciones geométricas heredadas de los griegos, los cuales no disponían de métodos generales para el trazado de rectas tangentes a cualquier curva, ni mucho menos relacionarla con la cuadratura de la curva. Tanto en el planteamiento como en las soluciones de las situaciones-problemas, se utilizó un lenguaje propio de la geometría euclidiana y sus argumentos eran puramente sintéticos (PINO-FAN, 2014). En las soluciones propuestas, intervenían conceptos tales como curva continua creciente, ordenada, 
perpendicularidad, puntos, rectángulo, longitud, paralelismo. Así mismo, en la lección X planteada por Barrow, aparecen propiedades utilizadas siglos atrás por los griegos, como la perpendicularidad entre curvas, rectas que unen intersecciones opuestas al trazo de tangentes en figuras cónicas, entre otras. Con respecto a los procedimientos son los característicos de los matemáticos de la época: geométricos.

El problema resuelto por Barrow, abordado desde los griegos, al igual que por otros matemáticos de la época, forma parte de un sistema de prácticas al cual hemos denominado Tangentes-Cuadraturas, la cual reúne las prácticas matemáticas geométricas que, en dicha etapa histórica, conferían un significado parcial a la antiderivada.

\subsection{Configuración epistémica 2 (CE2): el problema de la relación fluxiones-fluentes}

Este problema, descrito en el apartado 3.3.1, es parte de un sistema de prácticas, que se ha denominado Fluxiones-Fluentes. Para describir los elementos de esta segunda configuración, consideramos dos problemas prototípicos propuestos por Newton (1736, p.19):

I. Dada la longitud del espacio descrito continuamente (es decir, en todo momento); encontrar la velocidad del movimiento en cualquier momento propuesto.

II. Dada la velocidad del movimiento continuamente; encontrar la longitud del espacio descrito en cualquier momento propuesto.

Estos problemas, y su solución, están basados en la concepción cinemática del movimiento continuo, y se utilizan dos conceptos centrales. El primero es el de fluente, entendido como cantidad de movimiento que varía respecto del tiempo. El segundo es el de fluxión, que es la velocidad de cambio del movimiento respecto del tiempo.

El primer problema se aborda desde el ejemplo propuesto por Newton (1736, p. 21): "Dada la curva de ecuación $\mathrm{x}^{3}-\mathrm{ax}^{2}+\mathrm{axy}-\mathrm{y}^{3}=0$, calcular las fluxiones".

Para resolver este problema, Newton sustituye en la ecuación dada $x$ y $y$ por $x+\dot{x} o$ y $y+\dot{y} o$ respectivamente, obteniendo la ecuación (PINO-FAN; GODINO; FONT, 2011):

$$
\begin{aligned}
\left(x^{3}+3 \dot{x} o x^{2}\right. & \left.+3 \dot{x}^{2} o^{2} x+\dot{x}^{3} o^{3}\right)-\left(a x^{2}+2 a \dot{x} o x+a \dot{x}^{2} o^{2}\right) \\
& +\left(a x y+a \dot{x} o y+a y \dot{o} x+a \dot{x} \dot{y} o^{2}\right)-\left(y^{3}+3 \dot{y} o y^{2}+3 \dot{y}^{2} o^{2} y+\dot{y}^{3} o^{3}\right)=0
\end{aligned}
$$

Luego, elimina $x^{3}-a x^{2}+a x y-y^{3}$, que es igual a cero (de acuerdo con la ecuación dada originalmente), divide después por un intervalo de tiempo infinitamente pequeño, notado $o \mathrm{y}$, por último, desprecia finalmente los términos en que, todavía, figura el factor $o$, quedando la ecuación: $3 \dot{x} x^{2}-2 a \dot{x} x+a \dot{x} y+a \dot{y} x-3 \dot{y} y^{2}=0$.

Se puede observar que Newton introduce nuevos conceptos/definiciones en sus desarrollos sobre el cálculo infinitesimal y, con ellos, nuevas expresiones de términos y 
notación (además de lenguaje algebraico, geométrico y descriptivo), entre los cuales coincidiendo con Pino-Fan (2011), podemos señalar los siguientes: a) $o$ es un intervalo de tiempo infinitamente pequeño; b) momento de $x$ que define como un incremento infinitesimal de $x$, que representa con $o x$ (análogamente define el momento de $y, o y$ ); c) En palabras de Newton (1736, p. 20): "Llamaré cantidades fluentes, o simplemente fluentes, a estas cantidades que considero aumentadas gradualmente e indefinidamente; las representaré mediante las últimas letras del alfabeto $v, x, y, z$, para distinguirlas de las otras cantidades"; d) El concepto fluxión definido con sus palabras: "representaré con las mismas últimas letras coronadas con un punto $\dot{v}, \dot{x}, \dot{y}, \dot{z}$, las velocidades con que las fluentes aumentan por el movimiento que las produce y que, por consiguiente, se pueden llamar fluxiones" (NEWTON, 1736, p. 20); e) Momento de la fluente es la cantidad infinitamente pequeña que varía una fluente como $x$ en un intervalo de tiempo infinitamente pequeño $o$, es decir, $\dot{x} o$. El método de las fluxiones de Newton es, a su vez, el procedimiento de esta configuración.

Para abordar el segundo problema Newton sugiere: "Sea la ecuación propuesta por $3 \dot{x} x^{2}-2 a \dot{x} x+a \dot{x} y-3 \dot{y} y^{2}+a \dot{y} x=0$, calcular las fluentes" (NEWTON, 1736, p. 26).

Sus escritos muestran la solución a este problema, indicando que se debe proceder de manera contraria a la sustitución del primer problema planteado, en el cual hace la sustitución de $x$ y $y$ por $x+\dot{x} o$ y $y+\dot{y} o$ respectivamente. Se procede, indica, separando la ecuación como se muestra a continuación: separar la expresión en dos partes donde se muestren tres términos en cada una, la primera $3 \dot{x} x^{2}-2 a \dot{x} x+a \dot{x} y$, y la segunda $-3 \dot{y} y^{2} *+a \dot{y} x$, esto para efectuar operaciones sobre cada expresión por separado. La primera parte se divide por $\frac{\dot{x}}{x}$ obteniendo $3 x^{3}-2 a x^{2}+a x y$. La segunda expresión se divide por $\frac{\dot{y}}{y}$, con lo que se obtiene $-3 y^{3} *+a x y$. Las nuevas expresiones se dividen por $3 \cdot 2 \cdot 1$, simultáneamente y se obtiene $x^{3}-a x^{2}+a x y-y^{3} *+a x y$. Por lo tanto la suma es, $x^{3}-a x^{2}+a x y-y^{3}=$ 0 , esta expresión es la relación necesaria de las cantidades $x$ e $y$.

En este último ejemplo propuesto por Newton, al igual que en el problema propuesto al inicio, vemos como argumenta los procedimientos (y en general sus definiciones y proposiciones) con base en consideraciones dinámicas y de infinitesimales, apoyándose siempre en el álgebra y el análisis geométrico.

Dentro de las proposiciones/propiedades dadas por Newton, podemos mencionar las siguientes: a) Si $a x^{m / n}=y$, entonces el área será $z=\frac{n}{m+n} a x^{m+n / n}$; b) Supongamos una curva cuya área está dada por la expresión $z=a x^{m}$, donde $m$ es entero o fraccionario 
entonces la curva está dada por la expresión $y=\max ^{m-1}$ (derivación); c) Dada una $y=$ $\max ^{m-1}$ entonces el área comprendida bajo la curva es $z=a x^{m}$ (integración).

En cuanto a los tipos de situaciones/problemas de esta configuración, Newton abordó problemas sobre el cálculo de la velocidad del movimiento en un tiempo dado cualquiera, dada la longitud del espacio descrito, y viceversa.

El hecho de que sus algoritmos hayan sido aplicado a muchos problemas, como por ejemplo, método para encontrar mínimos en funciones o el método para encontrar aproximaciones de raíces de una función lineal, entre otras, hace que esta configuración sea altamente general o intensiva (PINO-FAN, 2014). Así, vemos como en esta configuración las fluxiones o velocidades de los movimientos de las fluentes - que en la actualidad conocemos como la derivada - y las fluentes o cantidades que varían respecto al tiempo - que en la actualidad conocemos como antiderivación - son entendidos por Newton como procedimientos recíprocos.

\subsection{Configuración epistémica 3 (CE3): el problema de la relación diferenciales- sumatorias}

Este problema, descrito en el apartado 3.3.2, planteado por Leibniz, forma parte de un sistema de prácticas que se ha denominado Sumatoria-Diferencial. Este sistema de prácticas lo analizamos a partir de dos problemas prototípicos. El primero está basado en la construcción del cálculo diferencial a partir de las diferencias infinitesimales, mientras que el segundo se basa en la construcción del cálculo sumatorio (cálculo integral) a partir de la suma de diferencias infinitamente pequeñas.

Leibniz abordó situaciones/problemas sobre máximos y mínimos, tangentes y puntos de inflexión; esto se evidencia claramente en el título de su obra Nova methodus pro maximis et minimis, intemque tangetibus, qua nec irrationales quantitates moratur (Nuevo método para los máximos y mínimos, así como para las tangentes, el cual puede también aplicarse a las cantidades fraccionarias e irracionales), en la cual introduce, por primera vez, la expresión cálculo diferencial y proporciona las fórmulas que hasta ahora conocemos para derivar productos, cocientes, potencias y raíces, todas éstas acompañadas de aplicaciones geométricas tales como la búsqueda de tangentes, máximos y mínimos, y de los puntos de inflexión.

Leibniz aborda tres ideas principales en el desarrollo del cálculo infinitesimal, así lo explica Bos (1984), la primera de ellas es la idea filosófica de characteristica generalis (una característica general), la segunda se refiere a las sucesiones de diferencias, y la tercera idea 
principal, fue el uso del triángulo diferencial en las transformaciones de cuadraturas.

De acuerdo con Pino-Fan (2014), Leibniz utiliza un lenguaje simbólico general mediante el cual se pueden escribir con símbolos y fórmulas todos los procesos de argumentación y de razonamiento. Con este fin, logró introducir un nuevo lenguaje accesible, que aún se mantiene en nuestros días, el cual facilita la manipulación de los conceptos, procedimientos y argumentos, en el cálculo diferencial. Por ejemplo, Leibniz introduce los símbolos $\int \mathrm{y} d$, los cuales concibe como operadores que representan, respectivamente, la suma de rectángulos o suma de áreas y las diferencias entre dos valores sucesivos de $x$ o $y$. Así mismo, denota con $d x$ y $d y$ a las diferenciales de las variables $x$ e $y$ respectivamente, es decir, diferencias infinitamente pequeñas de $x$ e $y$.

Los lenguajes, nuevos términos y notaciones, introducidos por Leibniz, estaban asociados a una serie de nuevos conceptos/definiciones o procedimientos, primordiales en su cálculo de diferencias. Por ejemplo, $d y$ la utiliza para denotar el concepto de diferencial de una variable $y$, la cual define como la diferencia infinitamente pequeña entre dos valores sucesivos de $y$ (análogamente define la diferencial de la variable $x, d x$ ). Así mismo, como ya señalamos anteriormente, introduce el símbolo $\int$ para representar el proceso de sumar rectángulos o áreas de rectángulos, y $d$ para representar las diferencias entre dos valores sucesivos de $x$ o $y$. Las fórmulas proporcionadas por Leibniz, que hasta ahora conocemos, para derivar productos, cocientes, potencias y raíces, así como la regla de integración por partes, son ejemplos de proposiciones y procedimientos al mismo tiempo.

Leibniz concibe una suma tal como $\int y d y$ (que más tarde los Bernoulli llamarían integral) como la suma de los rectángulos infinitamente pequeños de base $d x$ y altura $y$; por lo tanto, la cuadratura de una curva es igual a $\int y d x$.

Así mismo, afirmaba que el proceso de integración, referido como proceso de sumación es inverso al proceso de diferenciación. Pronto se interesaría en la relación que existe entre $d x$ y $d y$, y del significado de expresiones tales como $d(u v), d(u / v)$, etc. Así, en un manuscrito fechado el 26 de junio de 1676, y titulado Methodus Tangentium Inversa (Método inverso de tangentes), Leibniz afirmaba que la mejor manera de encontrar las tangentes es determinando el cociente $\frac{d y}{d x}$, dándose cuenta de que la determinación de la tangente a una curva depende de la razón de las diferencias de ordenadas y abscisas cuando se hacen infinitamente pequeñas.

Uno de los resultados geométricos de Leibniz, equivalente a los hallazgos de Barrow, se obtiene cuando relaciona la tangente de una curva con la cuadratura de la misma (Figura 5). 
Él lo describe como sigue:

Sea AC una forma curva cuyo eje ordenado es $\mathrm{AB}$, el área de esta curva es llamado 1. Sea AD otra forma curva con el mismo eje de ordenado, este segmento de ordenada es BD, llamado "y”. Sea esta curva AD tal que el área ABC está dada por todas las eles ( $\mathrm{l}^{\prime} \mathrm{s}$ ) o escrito de otra forma $\int \mathrm{l}$, que es igual al producto de BD y una línea fija igual a ay; luego, tomando $\mathrm{B}(\mathrm{B})$ igual a la unidad, tenemos que $\mathrm{l}=\mathrm{aw}$, donde $\mathrm{w}: \mathrm{B}(\mathrm{B})=\mathrm{DB}: \mathrm{BT}$ o $\mathrm{w}=\mathrm{y} / \mathrm{d}, \mathrm{l}=\mathrm{ay} / \mathrm{d}$ (LEIBNIZ, 1920, p. 180).

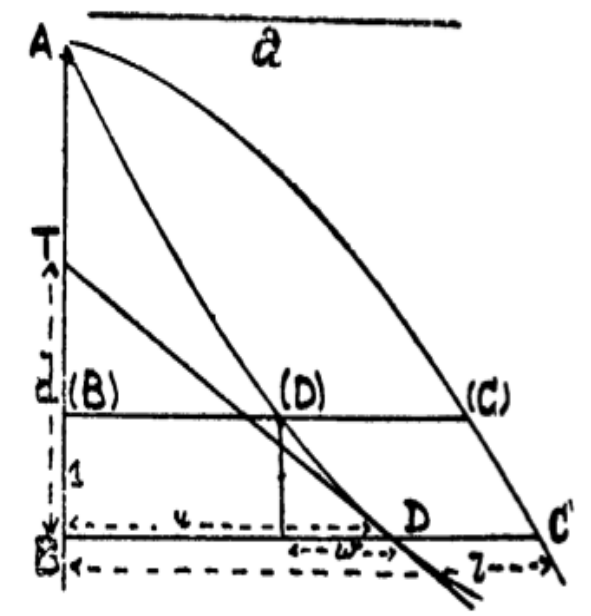

Figura 5 - Relación entre tangentes y cuadraturas (LEIBNIZ, 1920, p.180).

\subsection{Configuración epistémica 4 (CE4): el problema de la identificación de funciones elementales}

Este problema se aborda anteriormente en el apartado 3.4, y corresponde a uno de los sistemas de prácticas que identificamos y que lleva vinculada la configuración epistémica cuatro (CE 4) que se ha denominado funciones elementales. Caracterizamos este sistema de prácticas mediante un problema prototípico propuesto por Euler y construido sobre los problemas y soluciones de Leibniz. Como estudiamos anteriormente, fue Euler quien determinó la adición de una constante de integración al encontrar las función primitiva (antiderivada) y que en la actualidad conocemos como la constante de integración en la solución de una integral indefinida, que representa una familia de funciones de una función que ha sido derivada. De esta forma, Euler diferencia la integral particular (integral definida) de la integral completa (integral indefinida), como lo describe a continuación en su texto:

Definición 6. La integral se dice completa, cuando se representa la función buscada con cada extensión y con una constante arbitraria. Pero cuando esa constante se ha determinado en cierta manera, debe ser llamada integral particular. Corolario. De ahí que para todo caso se exprese una sola integral completa; pero las integrales son capaces de proporcionar un número infinito de integrales particulares. Así, la integral completa de la diferencial $\mathrm{xdx}$ es $\frac{1}{2} \mathrm{xx}+\mathrm{C}$, pero las integrales particulares $\frac{1}{2} \mathrm{xx}, \frac{1}{2} \mathrm{xx}+1, \frac{1}{2} \mathrm{xx}+2$, etc., son una multitud infinita (EULER, 1770, p. 11).

Euler utiliza un lenguaje nuevo en las matemáticas al adicionar una constante 
arbitraria para expresar la solución de la integral completa, que hoy se conoce como la adición de la constante de integración para significar una función que representa la familia de funciones de una función al ser derivada. Sus argumentos para diferenciar la integral completa de la integral particular, llevan a lo que, hoy, conocemos como la antiderivada.

Los aportes de Euler (1770), determinan con definiciones el hecho de que no todas las funciones tengan antiderivada o primitiva, pero sí puedan integrase a través de series trabajadas por Leibniz, como lo describe a continuación:

"Definición 5. Si las funciones, que se buscan en el cálculo integral de una relación de los diferenciales, no se pueden mostrar en forma algebraica, entonces estas son llamadas trascendentes, ya que varias de ellas trascienden las competencias de análisis común" (EULER, 1770, p. 5).

Euler, con la anterior definición advierte la forma en que debe ser abordada una antiderivada de una función, esta referencia da lugar a las funciones elementales. Prosigue su descripción de la forma de cómo abordar las funciones que no son elementales, como sigue:

\begin{abstract}
Ahora, en el primer intento de resolver una integral, las funciones no deben buscarse inicialmente, primero debe tomarse como una función trascendental. A menudo sucede que una integral algebraica puede ser obtenida a través de operaciones hábiles. Si la función que se busca es trascendental, se debe considerar con cuidado si ésta puede ser reducida a funciones más simples, o expresada como una función logarítmica o de forma angular, en cuyo caso la solución algebraica puede ser comparada igualmente. Pero si esto no se logra, conviene investigar la forma más sencilla de las funciones trascendentales, hasta que se pueda reducir el integrando tratado, con el método más adecuado, con el fin que los valores más cercanos a las funciones a trascender se puedan producir.

Teorema. Todas las funciones que se encuentran a través del cálculo de una integral son indeterminadas, se requiere una determinación por la naturaleza de la cuestión, que suministren la solución (EULER, 1770, p. 10).
\end{abstract}

Con el teorema anterior, Euler indica la forma de abordar funciones a través del cálculo integral, indicando qué hacer cuando éstas no pueden ser expresadas en forma elemental. Esta indicación hace que encontremos la solución por métodos diferentes a los elementales (numéricos), haciendo que la función pueda ser integrable sin tener antiderivada, esto ocurre cuando hace referencia en el teorema a encontrar la solución de ellas (las integrales) por la naturaleza en cuestión que suministre la solución, haciendo alusión al uso de métodos numéricos para la solución.

Pero la importancia del aporte de Euler está en la diferencia que establece entre las nociones de integral completa -que conocemos hoy como integral indefinida o antiderivada e integral - que hace alusión a integrales definidas - las cuales se calculan de diversas formas entre las cuales podemos destacar el TFC. 


\section{Reconstrucción del significado holístico de la antiderivada}

La identificación, caracterización y análisis de los sistemas de prácticas que se abordaron y desarrollaron en la diversas etapas históricas, han resultado en una propuesta de reconstrucción del significado holístico (PINO-FAN; GODINO; FONT, 2011) de la antiderivada. Cada sistema de práctica tiene vinculada una configuración epistémica que esta compuesta de objetos matemáticos primarios (situaciones/problemas, elementos lingüísticos, procedimientos, conceptos/definiciones, proposiciones/propiedades, y argumentos). La herramienta que nos proporciona el EOS, conocida como configuración epistémica nos ha ayudado a identificar y describir estos elementos.

Cada una de estas configuraciones epistémicas lleva asociado un significado parcial para la antiderivada. En nuestro caso concreto, hemos identificado cuatro configuraciones epistémicas para la antiderivada: 1) Tangentes-Cuadraturas (CE1); 2) Fluxiones-Fluentes (CE2); 3) Sumatorias-Diferencias (CE3); 4) Funciones Elementales (CE4).

El esquema de la Figura 6 muestra los significados parciales identificados, cada uno de ellos asociado a su respectiva configuración epistémica. De igual forma, dicho esquema muestra la relación entre algunas configuraciones de acuerdo a su generalización, así como la ubicación de la configuración en una línea de tiempo de acuerdo con su desarrollo.

Si bien, cada configuración es única, el esquema de la Figura 6, muestra algunas conexiones entre configuraciones. Estas conexiones, descritas por una línea discontinua, indican que con los elementos de dicha configuración es plausible resolver algunas situaciones-problema de otra configuración.

En el caso particular de la cuarta configuración (CE4), es intensiva por sus conexiones a otros sistemas de practicas indistintamente del tiempo histórico en que se encuentren, dado que con los elementos de (CE4) se pueden resolver algún tipo de problemas de los sistemas de prácticas que llevan asociadas las configuraciones CE1, CE2 y CE3, respectivamente.

Por otra parte, el esquema de la Figura 6, muestra la evolución, respecto de los elementos y características, de las configuraciones epistémicas y, por ende, de los diversos significados de la antiderivada a través del tiempo. Lo anterior se indica con conexiones con líneas continuas. Esta evolución esta dada en el sentido de que una nueva configuración, toma elementos de una configuración establecida, dando paso a la nueva configuración. En nuestro caso, son las configuraciones CE2 y CE3, las que toman elementos establecidos en la primera configuración (CE1), para activar nuevos elementos en dichas nuevas configuraciones, indistintamente de que estas configuraciones estén establecidas en la misma etapa histórica. 
De la misma forma actúa la configuración (CE4), al tomar elementos de la configuración (CE3).

La consideración conjunta de situaciones-problemas asociadas a sus respectivas configuraciones epistémicas y sus relaciones de evolución y/o solución de problemas, ilustrados en el esquema en la Figura 6, es lo que conforma la propuesta de reconstrucción del significado holístico de la antiderivada.

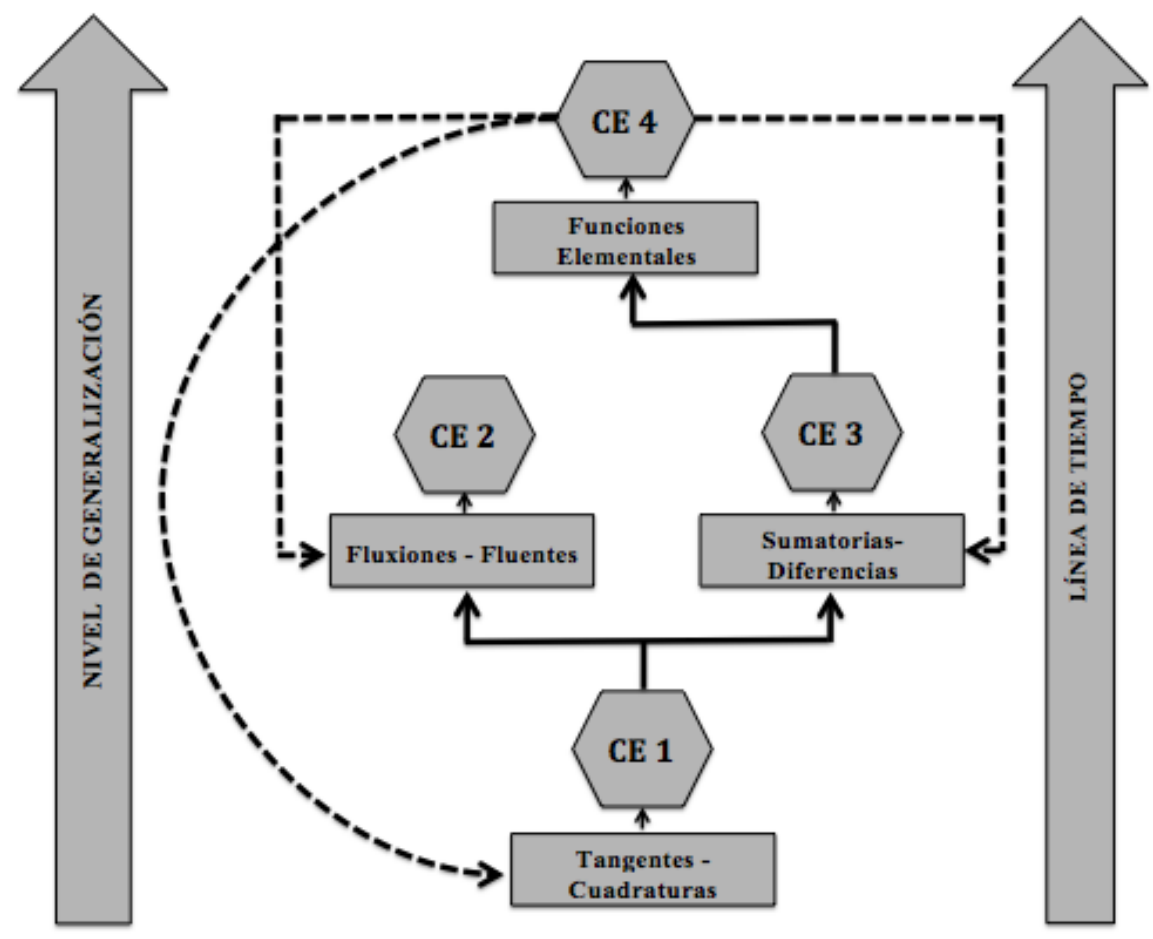

Figura 6 - Significado holístico de la Antiderivada

\section{Consideraciones finales}

La propuesta de reconstrucción del significado holístico de los objetos matemáticos, en general, resulta especialmente importante porque a través de ellos se identifican significados parciales de un objeto en la historia, los cuales constituyen los significados de referencia que los currículos de matemáticas deberían considerar para la comprensión de los objetos matemáticos. El análisis de problemáticas por medio de la herramientas del EOS, en particular de la noción de configuración epistémica (PINO-FAN; GODINO; FONT, 2011), nos ha permitido describir, de forma sistemática, situaciones/problemas, elementos lingüísticos, procedimientos, conceptos/definiciones, proposiciones/propiedades y argumentos, involucrados en las prácticas desarrolladas en diversos momentos históricos y que dieron paso al surgimiento y evolución de la antiderivada.

En este trabajo nos hemos propuesto realizar una reconstrucción del significado 
holístico para la antiderivada, con tal fin se realizó un recorrido histórico con el cual se identificaron problemáticas que dieron luces de la diversidad existente de prácticas desarrolladas, a propósito de la solución de situaciones-problemas, como lo fueron la relación entre la tangente de una curva y la cuadratura de la misma, así como la relación de las fluxiones con la fluentes y viceversa, entre otros.

El estudio resultó en la identificación de cuatro configuraciones epistémicas que se denominaron: 1) Tangentes-Cuadraturas (CE1); 2) Fluxiones-Fluentes (CE2); 3) SumatoriasDiferencias (CE3); 4)Funciones Elementales (CE4). Cada una de estas cuatro configuraciones epistémicas, a su vez, llevan asociado un significado parcial distinto para la antiderivada que van desde lo puramente geométrico hasta lo formal (CE4). De acuerdo con Pino-Fan (2014), y a los resultados de este estudio, la herramienta configuración ontosemiótica en su versión epistémica (o configuración epistémica) se prevé como una herramienta teórico-metodológica potente que permite determinar significados parciales para los objetos matemáticos, caracterizando prácticas matemáticas e identificando los objetos matemáticos primarios, y sus significados, involucrados en dichas prácticas.

El tipo de estudio histórico, como el desarrollado en este trabajo, es especialmente importante, coincidiendo con Doorman y Van Maannen (2008), por que permite dar indicaciones de cómo evoluciona una noción y su desarrollo conceptual.

\section{Reconocimientos}

Este trabajo ha sido realizado en el marco del proyecto de investigación Fondecyt de iniciación $\mathrm{N}^{\mathrm{o}} 11150014$, financiado por la Comisión Nacional de Investigación Científica y Tecnológica (CONICYT) de Chile.

\section{Referencias}

ANACONA, M. La Historia de las Matemáticas en la Educación Matemática. Revista EMA. Investigación e inovacion en educación matematica, Bogotá, v. 8, n. 1, p. 30-46, abr. 2003.

BARROW, I. Geometrical Lectures. Translated from the Latin Edition by Edmund Stone. Londres: Cambridge University. 1735.

BOS, H. J. M. Newton.Leibniz y la tradición leibniziana. In: GRATTAN-GUINNESS (Coord.). Del cálculo a la teoría de conjuntos, 1630-1910. Una introducción histórica. Traducción M. Martínez . . Madrid: Alianza Universidad. 1984. p. 69-124.

BOYER, C. The history the calculus and its conceptual developtmen. New York: Dover Publications, inc. 1959. 
CABAÑAS-SÁNCHEZ, G. El papel de la noción de conservación del área en la resignificación de la integral definida. Un estudio socioepistemológico. 2011. 455p. Tesis (Doctorado en Ciencias)Departamento de Matemática Educativa, Centro de Investigación y de Estudios Avanzados del Instituto Politécnico Nacional, México, 2011

CANTORAL, R. Desarrollo del pensamiento matemático. México: Trillas, 2000.

CANTORAL, R.; FARFÁN, R. Desarrollo conceptual del cálculo. Australia: Thompson.,2004.

COLLETE, J. Historia de las matemáticas II. México: Siglo XXI, 1985.

CORDERO, F. Reconstrucción de significados del cálculo integral: La noción de acumulación como una argumentación. México: Grupo Editorial Iberoamerica, 2002.

CRISOSTOMO, E. Idoneidad de Procesos de estudio del cálculo integral en la formación de profesores de matemáticas: Una aproximación desde la investigación en didactica del cálculo y el conocimiento profesional. 2012. 544 p. Tesis (Doctorado en Didáctica de la Matemática)- Facultad de Ciencias de la Educación, Universidad de Granada, Granada, España, 2012.

D’AMBROSIO, U. Priorizar história e filosofia da matemática. Cuadernos de investigación y formación en educación matemática, San José, v. 8, n. 11, p. 175-186, dic. 2013.

DOORMAN, M.; MAANNEN, J. V. A historical perspective on teaching and learning calculus. Australian Senior Mathematics Journal, Adelaide, v. 22, n. 2, p. 4-14. 2008.

EULER, L. Intitutionum Calculi Integralis. Translated from the Author's Latin Original. St. Petersburg. 1770.

GODINO, J. D.; BATANERO, C. Significado institucional y personal de los objetos matemáticos. Recherches en Didactique des Mathématiques, Paris, v. 14, n. 3, p. 325-355, 1994.

GODINO, J. D.; BATANERO, C. Clarifying the meaning of mathematical objects as a priority area of research in Mathematics Education. In: Sierpinska, A; Kilpatrick, J (Orgs.). Mathematics education as a research domain: A search for identity. Dordrecht: Kluwer, 1998. p. 177- 195.

GODINO, J. D.; BATANERO, C.; FONT, V. The onto-semiotic approach to research in mathematics education. ZDM. The International Journal on Mathematics Education, Dordrecht, v. 39, n. 1, p.127-135, mar. 2007.

LEIBNIZ, W. G. The early mathematical manuscripts of Leibniz. New York: Dover Publications, inc. 1920.

MATEUS, E. Epistemología de la derivada como fundamento del cálculo diferencial. Voces y silencios: revista latinoamericana de educación, Bogotá, v. 2, p. 3-21, dic. 2011.

NEWTON, I. Philosophiae naturalis principia mathematica. Translated from the Author's Latin Original. Londres, 1686.

NEWTON, I. The method of fluxions and infinite series. Translated from the Author's Latin Original by John Colson. Londres, 1736.

NIKOLSKI, S. M. Elementos de Análisis Matemático. Moscú: Editorial Mir, 1985. 
PINO-FAN, L. Conocimiento didáctico-matemático de los profesores sobre la derivada: clarificando los significados de la derivada desde la perspectiva de la enseñanza y el aprendizaje. España: Editorial Académica Española, 2011.

PINO-FAN, L. Evaluación de la faceta epistemica del conocimiento didáctico matemático de futuros profesores de bachillerato sobre la derivada. Granada: Universidad de Granada, 2014.

PINO-FAN, L.; GODINO, J. D.; FONT, V. Faceta epistémica del conocimiento didáctico-matemático sobre la derivada. Educação Matemática Pesquisa, São Paulo, Brasil v. 13, n. 1, p.141-178, ene. 2011.

PONCE, J. C. Isaac Barrow y su versión geométrica del teorema fundamental del cálculo. Números: Revista de Didáctica en Matemáticas. Las Palmas de Gran Canarias, Canarias, v. 83, p. 123-130, jul. 2013.

STEWART, J. Cálculo de una variable transendentes y tempranas. 6. ed. Mexico: Cengaje Lerning, 2013.

Submetido em Outubro de 2014. Aprovado em Abril de 2015. 